\title{
Multiple peripheral aneurysms of the posterior inferior cerebellar artery: Case report and review of the literature
}

\author{
Jin Li, ZhiGang Lan, Min He, Chao You \\ Department of Neurosurgery, West China Hospital, Sichuan University, 37 Guo Xue Xiang Street, Chengdu - 610 041, China
}

\author{
Address for correspondence: \\ Dr. Min He, \\ Department of Neurosurgery, \\ West China Hospital, Sichuan \\ University, 37 Guo Xue Xiang Street, \\ Chengdu, Sichuan - 610041 , \\ China. \\ E-mail: hxswjim@163.com
}

PMID: 19934574

DOI: $10.4103 / 0028-3886.57802$

\begin{abstract}
Multiple peripheral aneurysms of the posterior inferior cerebellar artery (PICA) are extremely rare. We describe a patient with four distinct aneurysms in the peripheral segments of the left PICA succefully treated surgically with an excellent outcome. Pathological examination of the excised aneurysmal sac showed no evidence of infectious etiology.
\end{abstract}

Key words: Cerebral aneurysm, multiple aneurysms, posterior inferior cerebellar artery

\section{Introduction}

Aneurysms of the posterior inferior cerebellar artery (PICA) are uncommon and account for only $0.5 \%$ of all intracranial aneurysms. ${ }^{[1]}$ These aneurysms usually occur at the origin of the PICA from the vertebral artery. Peripheral PICA aneurysms are relatively rare, most of them are saccular in shape and single in number. ${ }^{[2]}$ Multiple peripheral aneurysms of the PICA are rarer. We describe a patient with four distinct peripheral aneurysms of the left PICA. All aneurysms were microsurgically treated, with an excellent clinical outcome.

\section{Case Report}

A 61-year-old previously healthy woman was admitted to our hospital two days after sudden onset of headache and vomiting. There was no history of hypertension and smoking. On admission, she was drowsy and complained of severe occipital headache. Blood pressure was 135/85 mmHg. The neurological examination was unremarkable except for moderate neck stiffness. Computed tomography (CT) scan demonstrated blood in the fourth ventricle and no obvious ventricular dilatation [Figure 1]. Four-vessel cerebral angiography revealed four aneurysms in the peripheral segments of the left PICA with three located on the telovelotonsillar segment and one on the cortical segment [Figure 2]. There were no other cerebral aneurysms or vascular malformations. Erythrocyte sedimentation rate and total leukocyte count were normal. In view of peripheral location and multiplicity of these aneurysms, infectious etiology was suspected initially. Multiple blood cultures were negative for any pathogens.

A middle suboccipital craniectomy and C1 laminectomy were performed. When the dura mater was opened, an aneurysm $(5 \times 3 \mathrm{~mm})$ was easily identified arising from the cortical branch of the PICA just near the left tonsil [Figure 3]. The aneurysm was excised considering the more distal branch anastomosis. With gentle and gradual lateral retraction of the cerebellar tonsils, the roof and outlet of the fourth ventricle were explored. Three aneurysms with "string of beads" appearance were located deep in the outlet of the fourth ventricle. Complete exposure of the necks of all the three aneurysms was impossible because all the aneurysms were closely incorporated with PICA [Figure 4] making direct neck clipping impossible. We carefully excised all the three aneuryms without damaging the surrounding structures. The aneurysmal sacs were sent for pathological examination and culture to rule out an infectious etiology.

The postoperative course was uneventful. A postoperative angiography showed total obliteration of the four aneurysms [Figure 5]. The pathological examination and cultures showed negative for infection. The patient had a good recovery postoperatively. 


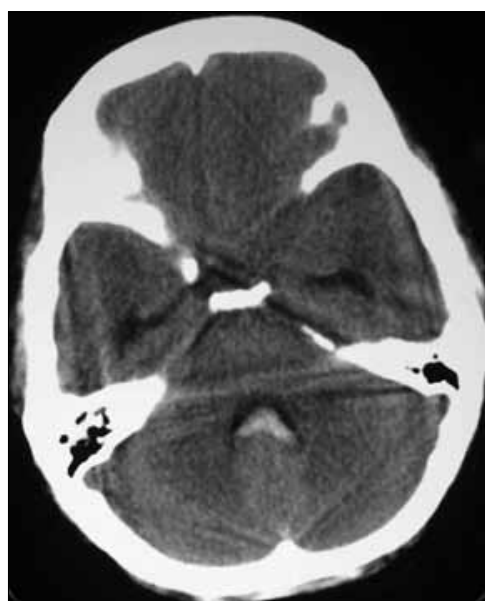

Figure 1: Preoperative axial plain computed tomography showing hemorrhage in the fourth ventricle

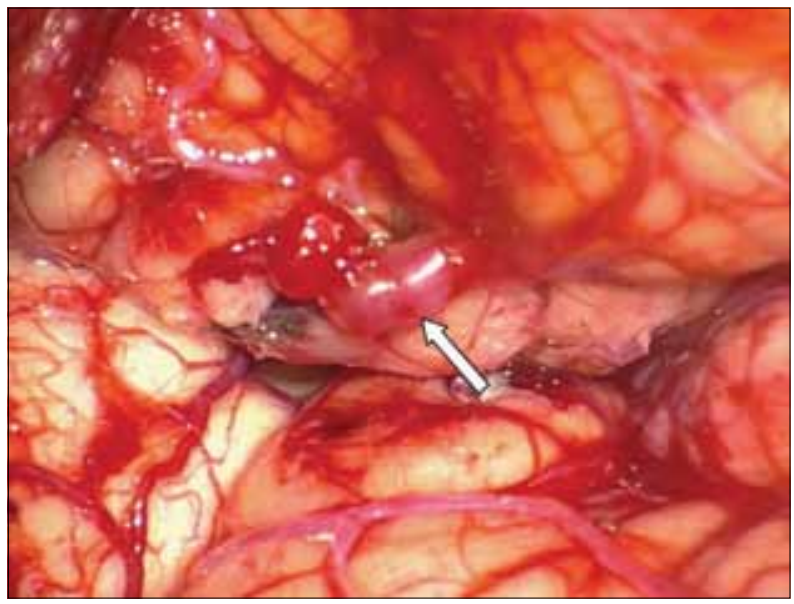

Figure 3: Intraoperative exposure showing the fourth aneurysm (arrow) located on the cortical segment of the left posterior inferior cerebellar artery

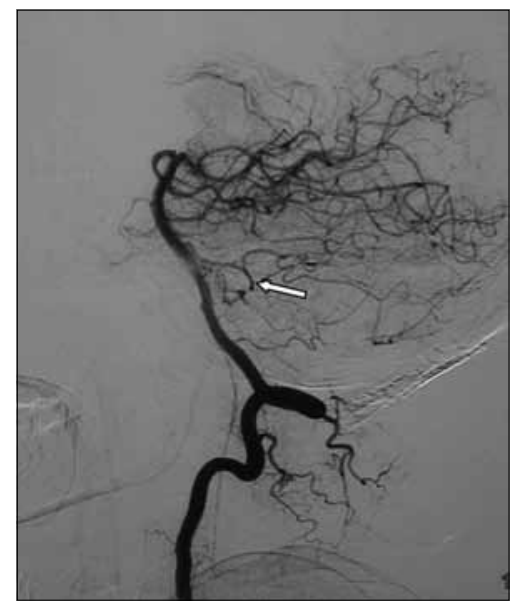

Figure 5: Postoperative lateral view of the left vertebral angiogram showing the complete obliteration of the aneurysms and preservation of the proximal portion of posterior inferior cerebellar artery (arrow)

\section{Discussion}

Aneurysms arising from the PICA are relatively uncommon, they usually occur at the junction of the vertebral artery and PICA. ${ }^{[1]}$ Distal aneurysms arising

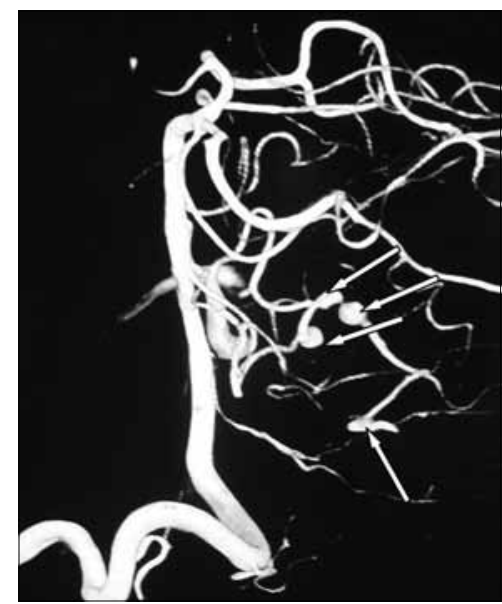

Figure 2: Preoperative lateral left vertebral three-dimensional angiogram demonstrating four aneurysms (arrows) in the left distal posterior inferior cerebellar artery

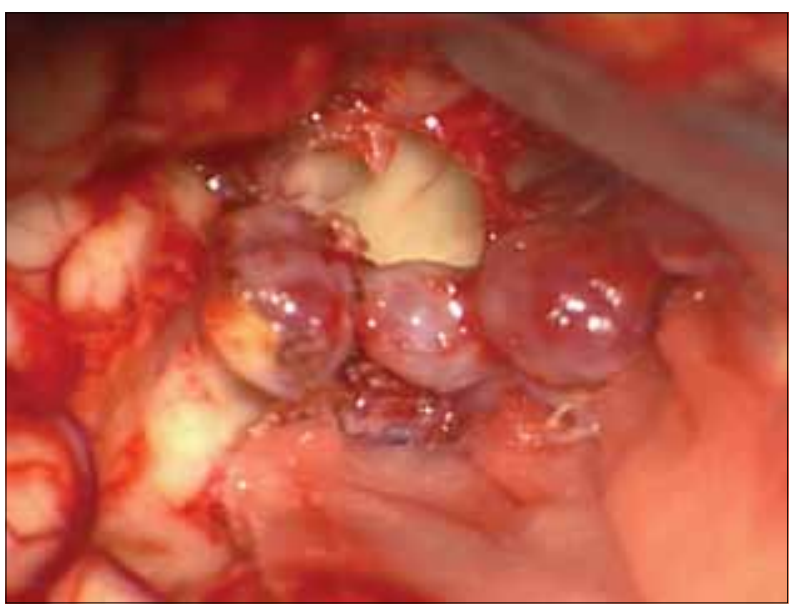

Figure 4: Intraoperative exposure showing three aneurysms lined in tandem in the telovelotonsillar segment of the left posterior inferior cerebellar artery

from more peripheral PICA segments is a very rare entity with an incidence of $0.28 \%$ to $1.4 \%$ of all cerebral aneurysms. ${ }^{[2]}$ Multiple aneurysms of the distal PICA are even rarer, and the first case was reported in 1982 by Hiscott $e t$ al. ${ }^{[3]}$ To date, only eleven cases of multiple peripheral aneurysms located on a single PICA have been reported in the literature [Table 1]. ${ }^{[3-10]}$ The gender distribution was eight women and three men. The average age was 54 years (range $27-68$ years). In the eleven patients reported, a total of thirty aneurysms were found and the number of aneurysms per artery ranged from two to five. Half of these aneurysms were located on the telovelotonsillar segment. All patients except one presented with subarachnoid hemorrhage (SAH). One of patients with multiple peripheral PICA aneurysms had associated with fibromuscular dysplasia (FMD). She was treated successfully by flow reversal therapy. ${ }^{[10]}$

The surgical approaches to multiple aneurysms of the distal PICA have been discussed ${ }^{[6,7,9]}$ It is important to know from 


\begin{tabular}{|c|c|c|c|c|c|c|}
\hline Author/date & Age/sex & Location & No. of aneurysms & Presentation & Treatment & Outcome \\
\hline Hiscott/1982 & $61 / F$ & R. 3.3.3 & 3 & Hemorrhage & Clipping & Good \\
\hline \multirow[t]{2}{*}{ Gacs/1983 } & $44 / F$ & L.1.3.3. & 3 & Hemorrhage & Clipping & Good \\
\hline & $64 / M$ & L.3.3. & 2 & Hemorrhage & Clipping & Good \\
\hline Yamamoto/1984 & $68 / F$ & L.4.4. & 2 & Hemorrhage & Clipping & Died \\
\hline Beyerl/1986 & $41 / F$ & L.1.1.3.4.4. & 5 & Hemorrhage & Clipping & Good \\
\hline Dernbach/1988 & $47 / M$ & R.3.3 & 2 & Asymptomatic & Clipping & Good \\
\hline Kitahara/2005 & $67 / F$ & $\mathrm{R} 2,2$ & 2 & Hemorrhage & Clipping & Good \\
\hline \multirow[t]{2}{*}{ Demirgil/2008 } & $62 / M$ & $\llcorner 4,4$ & 2 & Hemorrhage & Clipping & Good \\
\hline & $49 / F$ & $\mathrm{R} 4,4$ & 2 & Hemorrhage & Clipping & Good \\
\hline Kimura/2008 & $27 / F$ & $\mathrm{~L} 1,2,2$ & 3 & Hemorrhage & Embolization of left VA & Good \\
\hline Present case & $61 / F$ & L.3.3.3.4 & 4 & Hemorrhage & Excision & Good \\
\hline
\end{tabular}

PICA - Posterior inferior cerebellar artery; VA - Vertebral artery; PICA segments: 1 - anterior or lateral medullary; 2 - tonsillomedullary; 3 - telovelotonsillar; 4 - cortical

which segment the aneurysm arises. The application of either a midline suboccipital or a paramedian craniectomy will depend on the location of the aneurysms. ${ }^{[6]}$ When the aneurysms are located on the anterior or lateral medullary segment of the PICA, a lateral approach is best for the exposure because superomedial retraction of the cerebellum is easy with this approach. For more distal aneurysms, such as tonsillomedullary, telovelotonsillar and cortical segments of the PICA, a midline suboccipital craniectomy is preferable because exposure of the vermis and the fourth ventricle can easily be obtained.

Neck clipping of the aneurysm is still the best choice for the peripheral aneurysms of the PICA. ${ }^{[2,6]}$ For proximal aneurysms of the PICA, clipping of the aneurysm neck is essential because trapping of the aneurysm may compromise the vital perforating arteries to the brainstem. However, if necessary, trapping can be performed in the segments distal to the choroidal loop as there are no perforators to the brainstem or deep nuclei beyond this point. ${ }^{[6]}$ In our patient, three aneurysms were in a "string of beads" appearance and neck clipping was impossible, so we performed aneurysm excision. The fourth aneurysm was located on a more distal cortical branch. As no important artery arose from the parent vessel, excision was performed with good result.

We do not have any good explanation for the very unusual occurrence of the four discrete aneurysms located in a single PICA. Our initial suspicion was infectious etiology considering the peripheral location and multiplicity of these aneurysms. Septic embolus might infect the vascular wall and migrate distally causing several aneurysms. However, the cultures and pathological examinations were negative. Thus, it is unlikely that the aneurysms were infective in origin. Etiologies for multiple aneurysms such as FMD, polycystic kidney, polyarteritis nodosa and other vasculitis were also considered. However, the postoperative angiographic findings of aorta, cranial and renal arteries were normal; echocardiography did not reveal any specific findings; and the abdominal CT scan was also negative. Thus a detailed workup fairly excluded these possible etiologies in our patient. During the operation, the involved PICA seemed perfectly normal and the aneurysms were not significantly different in appearance from other saccular aneurysms. The hemodynamic factor was less likely as she was normotensive. Probaly in some patients congenital factors may play a role in the pathogenesis of distal PICA aneurysms. ${ }^{[9]}$ Yet the etiology in our patient with four aneurysms on a single PICA remains unclear.

\section{References}

1. Locksley HB. Report on the Cooperative study of intracranial aneurysms and subarachnoid hemorrhage. Section V. Part I: Natural history of subarachnoid hemorrhage, intracranial aneurysms and arteriovenous malformations. Based on 6368 cases in the cooperative study. J Neurosurg 1966;25:219-39.

2. Lewis SB, Chang DJ, Peace DA, Lafrentz PJ, Day AL. Distal posterior inferior cerebellar artery aneurysms: Clinical features and management. J Neurosurg 2002;97:756-6.

3. Hiscott P, Crockard A. Multiple Aneurysms of the Distal Posterior Inferior Cerebellar Artery. Neurosurgery 1982;10:101-2.

4. Gács G, Viñuela F, Fox AJ, Drake CG. Peripheral aneurysms of the cerebellar arteries. Review of 16 cases. J Neurosurg 1983;58:63-8.

5. Yamamoto I, Tsugane R, Ohya M, Sato O, Ogura K, Hara M. Peripheral aneurysms of the posterior inferior cerebellar artery. Neurosurgery 1984;15:839-45.

6. Beyerl BD, Heros RC. Multiple peripheral aneurysms of the posterior inferior cerebellar artery. Neurosurgery 1986;19:285-9.

7. Dernbach PD, Sila CA, Little JR. Giant and Multiple Aneurysms of the Distal Posterior Inferior Cerebellar Artery. Neurosurgery 1988;22:309-12.

8. Kitahara T, Yoneda H, Kato S, Kajiwara K, Akimura T, Suzuki M. Multiple aneurysms arising from the posterior inferior cerebellar artery with unknown etiology: Case report. Neurol Med Chir (Tokyo) 2005;45:523-5.

9. Demirgil B, Günaldi O, Tugeu B, Postalci L, Colluoglu B, Tanriverdi O, et al. Multiple aneurysms of the distal posterior inferior cerebellar artery: Two case reports. Minim Invasive Neurosurg 2008;51:249-52.

10. Kimura H, Hosoda K, Hara Y, Kohmura E. A very unusual case of fibromuscular dysplasia with multiple aneurysms of the vertebral artery and posterior inferior cerebellar artery: Case report. J Neurosurg 2008;109:1108-12.

Accepted on 02-07-2009

Source of Support: Nil, Conflict of Interest: None declared. 